\title{
Estilo de vida y su impacto en la situación de salud general y bucal del adulto mayor de la Casa del adulto mayor de Chorrillos
}

\author{
Life's style and its impact in general and oral health's status of elderly in elderly's house \\ of Chorrillos \\ Rosa Elizabeth Ysla-Cheé ${ }^{1 a}$, María Pareja-Vásquez $^{1 b}$, Jaime Carlos Porras-Cerrón ${ }^{2 c}$
}

\begin{abstract}
RESUMEN
Objetivo: Determinar el impacto del estilo de vida del Adulto Mayor en su salud general y bucal. Material y Método: Se realizó un estudio descriptivo, transversal en una muestra constituida por 44 adultos mayores, de ambos sexos, que acudían a la Casa del Adulto Mayor de Chorrillos, en Lima, Perú, noviembre de 2014. Se registraron datos sociodemográficos, se desarrolló una historia clínica odontológica y se aplicó un cuestionario sobre alimentación, salud general, actividad física e intelectual y hábitos de higiene bucal. Se establecieron puntuaciones equivalentes a estilo de vida muy saludable, saludable y poco saludable. Al hacer el examen bucal se aplicaron los índices CPOD, CPITN, Índice Gingival de Löe y Silness e IHOS. Los participantes firmaron un consentimiento informado. Resultados: Del total de adultos mayores examinados el $84.1 \%$ mostraron un estilo de vida "Poco saludable". Las enfermedades sistémicas más prevalentes fueron hipertensión arterial, problemas oculares, problemas osteoarticulares y diabetes. Se observó un CPOD alto, $19,98 \pm 6,45$ y al menos el $75 \%$ de adultos mayores requerían tratamiento periodontal. Únicamente el $11,6 \%$ de los participantes registraron buena higiene bucal. No se encontró asociación estadística entre el Estilo de Vida y el CPOD, Índice gingival de Löe Silness, CPITN, e IHOS, Chi cuadrado ( $p>0.05$ ). Conclusiones: El porcentaje de estilo de vida "poco saludable" es muy alto en esta población. También lo es, la presencia de caries y enfermedad periodontal. No se halló asociación entre el estilo de vida de los adultos mayores de la Casa del adulto Mayor de Chorrillos y su salud bucal y general.
\end{abstract}

Palabras clave: Adulto Mayor; Estilo de Vida; Salud Bucal; Conductas relacionadas con la salud; Índice periodontal. (Fuente: DeCS BIREME)

\begin{abstract}
Objective: To determine the impact of the Older Adult's lifestyle on their general and oral health. Material and Method: A descriptive, cross-sectional study was carried out in a sample made up of 44 older adults, of both sexes, who attended the Casa del Adult Mayor de Chorrillos, in Lima, Peru, November 2014. Socio-demographic data were recorded , a dental clinical history was developed and a questionnaire on diet, general health, physical and intellectual activity, and oral hygiene habits was applied. Equivalent scores were established for very healthy, healthy and unhealthy lifestyle. When doing the oral exam, the CPOD, CPITN, Loe and Silness Gingival Index and IHOS were applied. The participants signed an informed consent. Results: Of the total of older adults examined, $84.1 \%$ showed an "Unhealthy" lifestyle. The most prevalent systemic diseases were high blood pressure, eye problems, osteoarticular problems and diabetes. A high DMFT was observed, $19.98 \pm 6.45$ and at least $75 \%$ of older adults required periodontal treatment. Only $11.6 \%$ of the participants registered good oral hygiene. No statistical association was found between Lifestyle and DMFT, Löe Silness gingival index, CPITN, and IHOS, Chi square ( $p>0.05$ ). Conclusions: The percentage of "unhealthy" lifestyle is very high in this population. So is the presence of tooth decay and periodontal disease. No association was found between the lifestyle of the elderly in the House of the Elderly of Chorrillos and their oral and general health.
\end{abstract}

Key words: Aged; Life Style; Oral health; Health Behavior; Periodontal Index. (Source: MeSH NLM)

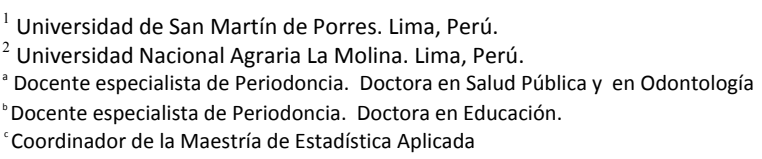

Citar como: Ysla Cheé RE, Pareja Vásquez M, Porras-Cerrón JC. R Estilo de vida y su impacto en la situación de salud general y bucal del adulto mayor de la Casa del adulto mayor de Chorrillos. KIRU. 2020; 17(4): 201-208. https://doi.org/10.24265/kiru.2020.v17n4.4 


\section{INTRODUCCION}

En los últimos años ha crecido el interés por el estudio del estilo de vida y su influencia en la salud de las personas. Más aun, cuando gran parte de la población mundial tiene estilos de vida poco saludables y esto puede repercutir no solo en la salud, sino también en la calidad de vida ${ }^{(1)}$.

Puede definirse al estilo de vida como la forma de vivir de las personas, basada en sus patrones individuales de conducta y su capacidad elegir, bajo la influencia de factores genéticos, socioeconómicos, culturales, económicos y medioambientales $^{(2)}$.

Se considera que el estilo de vida puede influir en la salud general y también en el desarrollo de enfermedades bucales ${ }^{(3,4)}$. Incluso la OMS sugiere que la promoción de la salud general y bucal se integren, considerando que ambas comparten factores de riesgo modificables (como dietas no saludables con alto contenido de azúcar, el consumo de tabaco y de alcohol) asociados a importantes enfermedades no transmisibles, como la hipertensión arterial, la diabetes, enfermedades respiratorias crónicas y el cáncer, ${ }^{(5)}$ que están relacionadas con el estilo de vida ${ }^{(4)}$.

Así mismo, debe tenerse en cuenta, que los problemas de salud bucal pueden alterar significativamente la calidad de vida de las personas, especialmente de los adultos mayores $\left({ }^{6-}\right.$ 9). Diversos factores como la falta de accesibilidad a la atención odontológica, costos de atención entre otros, pueden contribuir al deterioro de la función masticatoria, la deglución, la fonación y estética facial en esta población. A la vez, los daños ocasionados por las enfermedades bucales en este grupo de pacientes tienden a ser mayores y en su mayoría irreversibles ${ }^{(8)}$.

Diversos estudios sobre el estado bucal en esta población nos muestran un panorama en el cual se observan coincidencias sobre la pérdida dentaria, caries dental, enfermedad periodontal e higiene bucal deficiente. Sanchez Quintero Odalis (2017) ${ }^{(10)}$ mostró que aproximadamente la mitad de los adultos mayores de su estudio, tenían higiene bucal deficiente $(51,8 \%)$, sin diferencias significativas entre diabéticos y no diabéticos $(p=0,658)$, enfermedad periodontal en un $58,3 \%$, úlceras traumáticas en $13,3 \%$ queilitis angular en $7.9 \%$ y $59,1 \%$ de los pacientes tenía prótesis desajustadas. Brenes (2004) ${ }^{(11)}$, encontró una prevalencia de caries dental del $77,7 \%$, enfermedad periodontal del $52,9 \%$ y control de placa inadecuado en el $83,5 \%$, en una población de adultos mayores. Saez Carriera $(2007)^{(12)}$, encontró que el reborde alveolar reabsorbido fue el cambio bucal mas frecuente y que afectaba mayormente al sexo femenino. Además halló una relación directa entre los cambios bucales y las alteraciones sistémicas, principalmente en pacientes diabéticos.

Baskaradoss et al. $2019^{(4)}$, realizaron un estudio sobre la influencia del estilo de vida en el comportamiento de la salud dental, en 300 personas de un centro médico de Arabia Saudita. Los participantes fueron divididos en edad igual o menor de 32 años y mayores de 32 años. Hallaron que las personas con mejor estilo de vida tenían mejores hábitos de higiene bucal. Los adultos mayores tuvieron mejor control de salud bucal. Las mujeres fueron más regulares con sus controles dentales. Encontraron que el estilo de vida saludable se asociaba significativamente con comportamientos dentales positivos.

Gonzales R. y col. (2010) ${ }^{(13)}$ Realizaron un estudio en 352 adultos mayores en La Habana-Cuba, para determinar los factores de riesgo asociados con la aparición de lesiones bucales . Hallaron que el 60,7 $\%$ de los ancianos presentaron factores de riesgo; como la ingestión de alimentos condimentados y calientes $(83,4 \%)$, el hábito de fumar $(82,1 \%)$ el alcoholismo y el uso de prótesis, ambos con 72,4\%. Los más afectados fueron de sexo masculino y mayores de 80 años. El 48,5 \% presentaron lesiones bucales. Encontraron que predominaban los estilos de vida perjudiciales y la inadecuada educación para la salud ${ }^{(13)}$

García B et al.. (2012) ${ }^{(14)}$ Desarrollaron un estudio en 93 ancianos institucionalizados en Cienfuegos, Cuba, portadores de prótesis total. La presencia de lesiones bucales fue significativa en mayores de 80 años. El riesgo de desarrollar lesiones fue 2,98 veces superior en aquellos con una higiene bucal deficiente. Concluyeron que las lesiones bucales estuvieron muy relacionadas con el estado de conservación de la prótesis, higiene bucal, tiempo de uso de las prótesis; y con factores generales como la edad, enfermedades sistémicas (hipertensión arterial y diabetes) y uso de medicamentos.

En los últimos años se ha observado un aumento de la proporción de la población adulta mayor en la población peruana, de $5,7 \%$ en el año 1950 a $12,7 \%$ en el año $2020,{ }^{(15 t}$-por lo que es importante conocer las condiciones de salud bucal de los adultos mayores y como se relacionan con su estilo de vida. Este conocimiento permitirá promover estrategias orientadas a la prevención en la salud bucal y general del adulto mayor y tratamiento de las afecciones bucales prevalentes en esta población.

El objetivo del presente estudio es evaluar el estilo de vida en el adulto mayor y su impacto en su salud bucal y general. 


\section{MATERIAL Y MÉTODO}

Se realizó un estudio observacional descriptivo correlacional, prospectivo de corte transversal, en una población de adultos mayores de ambos sexos, con edades de 60 años a más, que acudían a "La Casa del Adulto Mayor" de Chorrillos en Lima, Perú, en el año 2014

La muestra estuvo conformada por 44 adultos mayores que acudían a esta institución y que aceptaron participar en el estudio. Se excluyeron a los edéntulos totales $y$ con trastornos motores 0 mentales que no les permitiera realizar una adecuada higiene bucal. La selección de la muestra fue por muestreo no probabilístico. Los participantes fueron agrupados en 4 grupos etarios: $60-65 ; 66-71 ; 72$ -77 y 78 a más.

Este estudio fue aprobado por el Comité de Ética en Investigación de la Facultad de Odontología de la Universidad de San Martín de Porres (Perú). Se explicó a los adultos mayores, cuales eran los objetivos y en que consistía el estudio y aquellos que aceptaron participar, firmaron un consentimiento informado.

Los investigadores llevaron a cabo un estudio piloto en un grupo de 20 participantes, con el fin de evaluar el nivel de comprensión de las preguntas y reproducibilidad del proceso.

Los datos se obtuvieron a través de un cuestionario y un examen estomatológico. Se registraron los datos sociodemográficos de los participantes, como edad y sexo y se continuó con la aplicación del cuestionario sobre el estilo de vida, que incluía preguntas acerca del: tipo de alimentación, actividad física, actividad intelectual, enfermedades sistémicas y hábitos de higiene bucal. Se clasificó el Estilo de vida como: muy saludable (51-56 puntos), saludable (51-56 puntos) y poco saludable (50 puntos o menos).

Para el examen estomatológico, se aplicó el Índice CPOD para evaluar dientes cariados, perdidos y obturados; el Índice Gingival de Loe y Sïlness y el Índice de Necesidades de Tratamiento Periodontal de la Comunidad (CPITN) para evaluar estado periodontal y el Índice de Higiene Oral Simplificado (IHO-S) de los doctores Greene y Vermillion para determinar la higiene bucal. Este examen fue realizado por una odontóloga especialista en periodoncia, quien examinó a cada participante sentado en una silla, con iluminación artificial adecuada, utilizando todos los implementos básicos para la mantener la bioseguridad, así como espejos bucales $\mathrm{N}^{\circ} 5$ y una sonda de la Universidad de Carolina del Norte (PUNC) Hu- Friedy (Estados
Unidos), debidamente esterilizados.

Los parámetros de calificación para el Índice Gingival de Löe y Sïlness fueron: 0) Encía normal, 1) Inflamación leve sin hemorragia, 2) Inflamación moderada con Hemorragia al sondeo y 3) Inflamación intensa con hemorragia espontánea.

Los códigos y criterios de calificación de la condición periodontal mediante el CPITN fueron: Código 0) periodonto sano, no necesita tratamiento, (Código 1) sangrado gingival que requiere mejorar la higiene bucal, (Código 2) presencia de cálculo dental, que requiere mejorar la higiene bucal y raspado profesional, (Código 3) bolsas periodontales de 4 a $5 \mathrm{~mm}$ que requiere mejorar la higiene bucal y raspado profesional y (Código 4) bolsas periodontales mayores de 6 $\mathrm{mm}$, que requiere mejorar la higiene bucal, raspado profesional y curetaje abierto o cirugía periodontal. Los valores del HIO-S, se registraron en códigos del 0-3.

Se aplicó el Coeficiente de Confiabilidad alfa de Cronbach, para determinar la confiabilidad de los instrumentos, así mismo las correlaciones entre los ítems y sus dominios.

Para el análisis estadístico, se utilizó el software estadístico SPSS versión 16.0. Se aplicó estadística descriptiva y se mostraron las frecuencias y porcentajes de las variables. Se aplicó la prueba no paramétrica de $U$ de MannWhitney para comprobar la diferencia entre muestras independientes. Se usó la prueba no paramétrica de chi cuadrado de Pearson para determinar la asociación entre el estilo de vida y los indicadores para medir la salud bucal y general. Se trabajó con un intervalo de confianza de $95 \%$ y un margen de error del $5 \%$. El valor de $\mathrm{p}<0,05$ se consideró estadísticamente significativo.

\section{RESULTADOS}

La muestra de la población de estudio estuvo conformada por un total de 44 adultos mayores, 25 mujeres $(56.8 \%)$ y 19 hombres $(43.2 \%)$, con una DV de \pm 6.59

Sobre el estilo de vida, los resultados mostraron que solo 07 participantes (15,9\%) presentaron un estilo de vida "saludable" y los 37 restantes "poco saludable" (84.1\%), ninguno se reportó un estilo de vida "muy saludable". Con respecto a la salud general, las enfermedades $\mathrm{O}$ condiciones sistémicas más frecuentes fueron: problemas 
oculares, hipertensión arterial, problemas osteoarticulares, estrés y diabetes (ver Tabla 1). La hipertensión arterial, fue ligeramente mayor en hombres $(n=11)$ que en mujeres $(n=8)$ siendo el grupo etario más afectado el de 72-77 años con el $20.5 \%$, seguido del grupo de 78 años a más, con el $13.6 \%$. Solo tres participantes no refirieron enfermedad alguna. Se encontró que el $27,3 \%$ de la muestra presentó tres enfermedades sistémicas y el $13,6 \%$ cuatro.

El estrés fue una condición muy observada, principalmente en el sexo femenino en una proporción de 3 a 1, con respecto a los hombres. Los grupos etarios más afectados por esta condición fueron los de 60 a 65 años y de 72 a 77 años.

Sobre la dieta, se encontró que había un mayor consumo de verduras, comparado al consumo de carbohidratos y frutas. Se observó que las mujeres tienen una dieta más saludable a base de verduras, y frutas, consumen una pieza de pan al día, muy poca azúcar, no comen entre comidas y no comen fuera de casa. Se hallaron diferencias significativas en el tipo de dieta de entre hombres y mujeres, $U$ de Mann-Whitney $p<0.05$

Con respecto a la actividad física, el $29,5 \%$ de los participantes de sexo masculino manifestaron tener actividades físicas y el $15,9 \%$ de participantes de sexo femenino, respondieron tener mayormente actividades manuales. Sobre la actividad intelectual, el $40.9 \%$ de participantes de sexo femenino manifestaron tener mucha actividad. El $15.9 \%$ de participantes de sexo masculino refirieron que preferían escribir.

El promedio del índice CPO fue de $19.98+6,45$, considerado muy alto según los estándares de la OMS , que lo considera elevado si es mayor a 18. Los dientes cariados y obturados registraron un promedio de 2.28 y 5, 20, respectivamente. Los dientes sanos tuvieron una media de 40.40. El componente dental perdido fue muy frecuente 14.80 .

Sobre la higiene bucal, se observó que solo el $11.6 \%$ presentó buena higiene, no se encontró asociación estadística entre el estilo de vida y el Índice de Higiene Oral (IHOS), Chi cuadrado $p>0.05$.

Con relación a la frecuencia del cepillado dental, un $43.2 \%$ de la muestra respondió que se cepillaba los dientes 2 veces al día. Sobre el acceso a educación en salud bucal, un 54\% manifestó estar enterado de las charlas sobre salud oral.

Tabla 1. Edad y enfermedades o condiciones sistémicas según sexo, de los adultos mayores de la Casa del adulto Mayor de Chorrillos

\begin{tabular}{|c|c|c|c|c|c|}
\hline \multirow{3}{*}{ Variables } & & \multicolumn{4}{|c|}{ Sexo } \\
\hline & & \multicolumn{2}{|c|}{ Mujeres } & \multicolumn{2}{|c|}{ Hombres } \\
\hline & & $\mathbf{n}$ & $\%$ & $\mathbf{n}$ & $\%$ \\
\hline \multirow{4}{*}{ Edad en años } & $60-65$ & 6 & $13.60 \%$ & 3 & $6.80 \%$ \\
\hline & $66-71$ & 4 & $9.10 \%$ & 3 & $6.80 \%$ \\
\hline & $72-77$ & 11 & $25.00 \%$ & 8 & $18.20 \%$ \\
\hline & $78 a+$ & 4 & $9.10 \%$ & 5 & $11.40 \%$ \\
\hline \multirow{6}{*}{$\begin{array}{l}\text { *Enfermedades/condiciones } \\
\text { sistémicas }\end{array}$} & Hipertensión arterial & 8 & $16 \%$ & 11 & $24.4 \%$ \\
\hline & Diabetes & 7 & $14 \%$ & 1 & $2,3 \%$ \\
\hline & Estrés & 9 & $18 \%$ & 3 & $6,6 \%$ \\
\hline & Problemas osteoarticulares & 10 & $20 \%$ & 6 & $13,3 \%$ \\
\hline & Problemas oculares & 13 & $26 \%$ & 15 & $33.3 \%$ \\
\hline & Problemas auditivos & 3 & $6 \%$ & 9 & $20 \%$ \\
\hline
\end{tabular}

* Algunos participantes tuvieron más de una enfermedad o condición sistémica

Tabla 2. Resultados del examen dental CPO: Componente cariado, ausente y obturado del Adulto Mayor de la Casa del Adulto Mayor de Chorrillos

\begin{tabular}{ccccccccccc}
\hline \multicolumn{3}{c}{ INDICE CPOD } & \multicolumn{3}{c}{ Cariados } & \multicolumn{3}{c}{ Perdidos } & \multicolumn{3}{c}{ Obturados } \\
$\bar{X}$ & DS & $\mathrm{n}$ & $\overline{\mathrm{X}}$ & $\mathrm{DS}$ & $\mathrm{n}$ & $\overline{\mathrm{X}}$ & $\mathrm{DS}$ & $\mathrm{n}$ & $\overline{\mathrm{X}}$ & $\mathrm{DS}$ \\
\hline 19,98 & 6.45 & 18 & $2.28+2,10$ & 44 & $14.80+8.13$ & 35 & $5.20+4.45$ \\
\hline
\end{tabular}


Al aplicar el CPITN, se halló que las condiciones más observadas fueron sangrado gingival (código 1) en un 18.9\% y cálculo dental (código 2) en un 19.3\%; correspondiéndoles como tratamiento, "Instrucción sobre higiene bucal" e "Instrucción sobre higiene bucal más raspado profesional", respectivamente. Se halló un $18.5 \%$ de sextantes calificados como sanos (Código 0). Un $5.3 \%$ de sextantes presentaron bolsas periodontales de 4 a $5 \mathrm{~mm}$. (Código 3). Menos del $1 \%$ presentaron bolsas mayores de $6 \mathrm{~mm}$. (Código 4). El sextante que presentó mayor presencia de cálculo dental fue el antero inferior y el que presentó mayormente la categoría de "sano" (código 1), fue el sextante antero superior. Se consideró 98 sextantes $(37.12 \%)$ como faltantes, porque no presentaron dientes para el índice.

Para determinar la condición gingival , se aplicó el índice gingival de Löe y Silness, se halló que el $75 \%$ de adultos mayores presentaron gingivitis moderada. El $39.5 \%$ de participantes de sexo femenino presentó gingivitis moderada y el $14 \%$ gingivitis severa. El 25,6\% de Adultos Mayores de 72-77 años presentó gingivitis moderada y el $11.6 \%$ entre $72-77$, gingivitis severa.

Con respecto a los hábitos de higiene bucal, los resultados mostraron que los adultos mayores de sexo femenino observaron mayor frecuencia de cepillado dental.

Tabla 3. Examen periodontal CPITN en Adultos Mayores de la Casa del Adulto Mayor de Chorrillos

\begin{tabular}{lcccccc}
\hline Criterios CPITN & $\begin{array}{c}\text { Sextante } \\
\text { superior } \\
\text { derecho }\end{array}$ & $\begin{array}{c}\text { Sextante } \\
\text { superior } \\
\text { anterior }\end{array}$ & $\begin{array}{c}\text { Sextante } \\
\text { superior } \\
\text { izquierdo }\end{array}$ & $\begin{array}{c}\text { Sextante } \\
\text { inferior } \\
\text { izquierdo }\end{array}$ & $\begin{array}{c}\text { Sextante } \\
\text { inferior } \\
\text { anterior }\end{array}$ & $\begin{array}{c}\text { Sextante } \\
\text { inferior } \\
\text { derecho }\end{array}$ \\
\hline 0 Sano & 7 & 16 & 7 & 6 & 11 & 2 \\
1 Sangrado & 7 & 6 & 8 & 9 & 9 & 11 \\
2 Cálculo dental & 4 & 3 & 8 & 8 & 18 & 10 \\
3 Bolsa de 4 a 5 mm & 4 & - & 2 & 2 & 1 & 5 \\
4 Bolsa mayor de 6 mm & 2 & - & - & - & - & - \\
\hline
\end{tabular}

Tabla 4. Relación entre las variables Índice de Higiene Oral Simplificado (IHOS) e Índice gingival deLöe Silness y el estilo de vida del Adulto Mayor de la Casa del Adulto Mayor de Chorrillos

\begin{tabular}{|c|c|c|c|c|c|c|}
\hline \multirow[b]{3}{*}{ Variables } & & \multicolumn{4}{|c|}{ Estilo de vida } & \multirow[b]{3}{*}{ valor de $\mathrm{P}$} \\
\hline & & \multicolumn{2}{|c|}{ Poco saludable } & \multicolumn{2}{|c|}{ Saludable } & \\
\hline & & $\mathrm{n}$ & $\%$ & $\mathrm{n}$ & $\%$ & \\
\hline \multirow{3}{*}{ IHOS } & Bueno & 4 & 9.3 & 1 & 2.3 & \multirow[t]{3}{*}{$p>0.05$} \\
\hline & Regular & 18 & 39,5 & 5 & 11,6 & \\
\hline & Malo & 15 & 35 & 1 & 2,3 & \\
\hline \multirow{4}{*}{$\begin{array}{l}\text { Índice gingival } \\
\text { de Löe } \\
\text { Silness }\end{array}$} & Sano & 1 & 2,2 & 0 & 0 & \multirow[t]{4}{*}{$p>0.05$} \\
\hline & Gingivitis leve & 1 & 2,2 & 1 & 2,2 & \\
\hline & Gingivitis moderada & 28 & 63,6 & 5 & 11,6 & \\
\hline & Gingivitis severa & 7 & 16 & 1 & 2,2 & \\
\hline
\end{tabular}

Con respecto a las dimensiones del estilo de vida ha habido diferencia significativa en los puntajes de alimentación entre hombres y mujeres, U de MannWhitney $p<0.05$. Se observó que el nivel de escolaridad e ingresos de los adultos mayores y su estilo de vida, no están asociados, chi cuadrado de Pearson $p>0.05$. No se encontró asociación entre el Estilo de Vida y CPOD, CPITN, IHOS y el Índice Gingival de Löe Silness, Chi cuadrado ( $p$ $>0.05$ )

\section{DISCUSIÓN}

El estilo de vida es sinónimo de hábitos o forma de comportamiento humano que se relaciona con diversos factores de su entorno y que incluso puede ser considerado como "causal de enfermedades" (4). Se considera que un estilo de vida saludable es básico para mantener una buena salud, por ello la OMS recomienda consumir una dieta balanceada (como mayor ingesta de frutas, verduras, pescado, cereales integrales y menos azúcar, grasas saturadas, sal, entre otros.) y realizar actividades físicas con regularidad ${ }^{(16,17)}$. 
Según la OMS, la salud general y bucal tienen factores de riesgo en común, que se relacionan con el estilo de vida ${ }^{(5)}$ (OMS XXI). Lo que coincide con resultados de investigaciones como las de Li et al. quien encuentra que llevar un estilo de vida saludable, se asocia con tener mas posibilidades de tener una vida libre de enfermedades crónicas como la diabetes, enfermedades cardiovasculares e incluso el cáncer. ${ }^{(18)}$

Además, es importante tener en cuenta que una buena salud bucal, le dará al individuo no solo un buen funcionamiento de su boca, sino también una apariencia estéticamente aceptable, lo que va a mejorar su calidad de vida ${ }^{(19)}$

Sobre la salud general de los adultos mayores Meneses Gómez ${ }^{(20)}$ encontró que esta afectada principalmente por enfermedades sistémicas crónicas, como la hipertensión arterial, la diabetes y problemas osteoarticulares, lo cual coincide con los resultados hallados en este estudio. Situación que es preocupante, dado que en los próximos años se estima que aumentará el porcentaje de la población adulta mayor y no se vislumbra una mejora, en sus condiciones generales de vida, de atención de salud, y promoción de estilos de vida saludable, lo que llevaría al incremento de la prevalencia de enfermedades crónicas no transmisibles, en este grupo poblacional ${ }^{(21)}$

En los problemas bucodentales: la caries dental medida con el índice CPOD tuvo un valor medio de 19.98 que corresponde a un nivel alto según los estándares de la OMS. Estos resultados coinciden con los de Bardales que refiere un CPO de 25.7 en 150 pacientes adultos mayores ${ }^{(22)}$

Singla $\mathrm{N}$ et al. ${ }^{(23)}$ halló en un estudio realizado en 800 sujetos con edades entre 20 y 50 años, que los participantes con nivel económico más bajo, de mayor edad, desempleados, de sexo femenino, con menor educación, menor frecuencia de cepillado dental, estilo de vida pobre o moderado, tienen mayor riesgo de tener caries dental. En este estudio se encontró que la mayoría de los participantes tenían un estilo de vida poco saludable y un CPOD muy alto, sin embargo no se halló asociación estadística entre el estilo de vida y la presencia de caries dental.

Baskaradoss et al. ${ }^{(4)}$ y López Castellanos et al. ${ }^{(8)}$, encontraron que los adultos mayores y en especial las mujeres, tuvieron comportamientos dentales positivos, resultados con los cuales coincide el presente estudio, en el cual se halló que los adultos mayores en un $43 \%$, realizaban el cepillado dental dos veces al día, y que las mujeres se cepillaban con mayor frecuencia que los hombres.
García B et al.. (2012) ${ }^{(14)}$ realizaron un estudio en 93 adultos mayores institucionalizados en Cienfuegos, Cuba, portadores de prótesis total. Encontraron que en los adultos mayores de 80 años la presencia de lesiones bucales fue muy alta y el riesgo de desarrollar lesiones fue 2,98 veces mayor en aquellos que tenían mala higiene bucal. Concluyeron que estas lesiones bucales estaban relacionadas con el estado de conservación y tiempo de uso de las prótesis, la higiene bucal y con otros factores como la edad, enfermedades sistémicas (hipertensión arterial y diabetes) y uso de medicamentos. No se encontraron coincidencias con los resultados de este estudio, en que no se halló relación entre las condiciones sistémicas y el estado bucal de los adultos mayores.

Gonzales R.et al. (2010) ${ }^{(13)}$ llevaron a cabo un estudio en 352 sujetos mayores de 60 años de La Habana-Cuba, año 2006, para determinar los factores de riesgo asociados con lesiones bucales. Encontraron que el $60,7 \%$ de los adultos mayores presentaron factores de riesgo como el consumo de alimentos muy condimentados y calientes $(83,4 \%)$, el hábito de fumar $(82,1 \%)$ el consumo de alcohol y el uso de prótesis $(72,4 \%)$. Los mayores de 80 años fueron los más afectados y principalmente los de sexo masculino. Encontraron que predominaban los estilos de vida perjudiciales y la inadecuada educación para la salud. Hubo coincidencias con este estudio con respecto a que predominan en el adulto mayor, los estilos de vida poco saludables $(74.1 \%)$ Sobre la educación para la salud, se observo una ligera diferencia, porque en el presente estudio hallamos que el $54 \%$ conocía sobre las charlas de salud bucal.

Rojas Gonzales et al. ${ }^{(24)}$ encontraron que los adultos mayores presentaban un mal estado periodontal, que el $100 \%$ necesitaba algún tipo de tratamiento, y el $82.3 \%$ requería tratamiento periodontal complejo. Se hallo coincidencias con sus resultados en cuanto al mal estado periodontal de los ancianos, y también que un alto porcentaje $(75 \%)$ requerían algún tipo de tratamiento, entre gingival $y$ periodontal, pero en este estudio se halló que menos del $7 \%$ necesitaban tratamiento periodontal complejo. Por otro lado, Pérez Hernández L. ${ }^{(25)}$ refiere que la población de adultos mayores que evaluó, presentaba enfermedad periodontal, principalmente en grado leve (63.2\%), Así mismo, Naimi-Akbar A. et al. (26) hallaron en su estudio en 1610 sujetos con edades entre 28 y 75 años, que el $31 \%$ tenían periodontitis de leve a moderada y el $6 \%$ periodontitis severa. 
Resultados similares a los obtenidos en este estudio en el cual predominaron los casos con sangrado gingival y cálculos dentales, según los criterios de calificación del CPITN.

Sobre la higiene bucal en los adultos mayores, Pérez Hernández L. ${ }^{(25)}$ halló que es deficiente, similar a lo obtenido en este estudio donde se observó que al aplicar el IHOS el $88 \%$ presentaba de regular a mala higiene bucal. Con respecto a la educación sobre salud bucal este autor encontró un nivel de conocimientos predominante inadecuado (71.18\%), frente a los resultados de este estudio, en el cual se encontró un porcentaje algo mas favorable, en el cual el $54 \%$ de los participantes refirieron tener conocimiento sobre las charlas de salud bucal.

Como limitaciones de este estudio se puede citar, que el tamaño de la muestra es reducido comparado con otras investigaciones y que se recoge de una sola institución. Así mismo, que no fue posible realizar el muestreo al azar, debido a que la población no era muy numerosa. Además, se hallaron muy pocos estudios que contrasten el estilo de vida con la salud bucal de adultos mayores.

Se concluye que el estilo de vida que presentan los Adultos Mayores de la Casa del Adulto Mayor de Chorrillos es en su mayoría poco saludable (84.1\%).

Los adultos mayores de sexo femenino tuvieron una dieta mas saludable, mejor salud general y actividad intelectual más activa que los de sexo masculino. Los varones registraron mayor actividad física que las mujeres.

La salud general se ve afectada por la hipertensión arterial, diabetes, problemas oculares, enfermedades osteoarticulares y estrés.

Sobre la salud bucal, se halló un CPO muy alto, mayor que el recomendado por la OMS a la edad de 65 años. La higiene bucal de los adultos mayores es regular en un $51 \%$ y mala en un $37 \%$.

Sobre los hábitos de higiene bucal, el sexo femenino presentó mayor frecuencia de cepillado dental. El estilo de vida de los adultos mayores no esta asociado al índice CPO, CPITN, Índice de Löe y Silness y el IHOS. No hay asociación entre el estilo de vida de los adultos mayores y su salud general y bucal.

Se recomienda habilitar programas de promoción y prevención de salud general y bucal, dirigido a adultos mayores en instituciones publicas y privadas, organizaciones sociales, vecinales y comunales; que incluya orientación para tener estilos de vida más saludables y se resalte la importancia del autocuidado de la salud ${ }^{(26,27)}$.
Se requiere ampliar la cobertura de los servicios de salud, para mejorar la salud bucal y general, en esta población.

Contribuciones de autoría: REYCH, MCPV diseñaron el estudio, participaron en la recolección, análisis y procesamiento de datos. JCPC participó en el análisis y procesamiento de la información. Los autores redactaron y aprobaron la versión final del artículo.

Fuente de financiamiento: El estudio fue financiado por los autores.

Conflicto de intereses: Los autores declararon no tener conflictos de interés.

\section{REFERENCIAS}

1. Farhud DD. Impact of Lifestyle on Health. Iran $J$ Public Health. 2015;44(11):1442-1444.

2. Guerrero Montoya LR, León Salazar AR. Estilo de vida y salud. Educere [Internet].2010 [Citado 2020 abril 20];14(48):13-19. Disponible en:https:www.redalyc.org/articulo.oa?id=3561672 0002

3. Akhtar Hussain Bokhari. Lifestyle impact on oral health. S. Pak J Med Sci 2006 ;22 (3):336-337.

4. Baskaradoss, J. K, Geevarghese, A, Al-Mthen, A, Al-Ghamdi, H, Al-Haudayris, R, Al-Obaidy, S \& AlSaadi W. Influence of Lifestyle on Dental Health Behavior. Journal of lifestyle medicine[Internet]. 2019; [Citado 2020 abril 20]9(2):119124.Disponible https://doi.org/10.15280/jlm.2019.9.2.119

5. Oral health [Internet] World Health Organization; 2020 [Citado 2020 jul 10] Disponible en: https://www.who.int/es/news-room/factsheets/detail/oral-health

6. Diaz-Reissner Clarisse Virginia, Casas-García Irma, Roldán-Merino Juan. Calidad de Vida Relacionada con Salud Oral: Impacto de Diversas Situaciones Clínicas Odontológicas y Factores Socio-Demográficos. Revisión de la Literatura. Int.J. Odontostomat. [Internet]. $2017 \mathrm{Abr}$

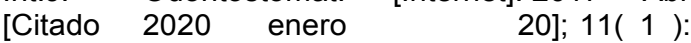
31-39. Disponible en:https://scielo.conicyt.cl/scielo.php?script=sci_a $r$ ttext\&pid=S0718-381X2017000100005\&Ing=es.

7. Rodríguez Fuentes Marcilia, Arpajón Peña Yunier, Herrera López Isis Bárbara. Autopercepción de salud bucal en adultos mayores portadores de prótesis estomatológica. Rev haban cienc méd [Internet]. 2016 Feb [Citado 2019 mayo 10] ;15(1).Disponible en: http://scielo.sld.cu/scielo.php?script=sci_arttext\&p i d=S1729-519X2016000100007\&lng=es.

8. López Castellanos G , Lopez Ramirez L, Nachon Garcia MG , Hernandez Lunagomez D. Salud bucal, salud bucal percibida y calidad de vida en adultos mayores. Rev Med UV, Julio - Diciembre 2014.

9. Sánchez Nurguiendo M., Román Velásquez M., Dávila Mendoza R., González Pedraza Avilés A. Salud Bucal en pacientes Adultos Mayores y su asociación con la calidad de vida. Revista de Especialidades Médico-Quirúrgicas. 2011;16 (2): 110-115 
10. Sánchez Quintero Odalis, Pérez Borrego Amparo, Fonseca Fernández Yenileidy, Cepero Santos Aimée, Calzadilla Mesa Xiomara María, Bertrán Herrero Grethell. Influencia de la Diabetes mellitus en la salud bucal del adulto mayor. Rev haban cienc méd [Internet]. 2017 Jun [Citado 2019Mayo 10] ; 16( 3 ): 361-370. Disponible en: http://scielo.sld.cu/scielo.php?script=sci_arttext\& pi $d=S 1729-519 \times 2017000300007 \&$ Ing=es

11. Brenes W, Hoffmaister F. Situaciones de salud bucal en personas adultas mayores: problemas y desafíos. Rev. costarric. cienc. méd [Internet]. 2004 Dec [Citado 2019 May 15]; 25(3-4):2741.Disponible en:

http://www.scielo.sa.cr/scielo.php?script=sci artt ext\&pid=S0253-29482004000200003\&lng=en

12. Sáez Carriera $R$, Carmona $M$, Jiménez Quintana Z, Alfaro X. Cambios bucales en el adulto mayor. Rev Cubana Estomatol [Internet]. $2007 . \quad$ Dic [Citado 2020 febrero 15] ; 44( 4 ): . Disponible en: http://scielo.sld.cu/scielo.php?script=sci_arttext\& pi $d=S 0034-75072007000400011 \&$ Ing=es.

13. Gonzáles Ramos. Herrera I. Osorio M. Madrazo D. Principales lesiones bucales y factores de riesgo presentes en población mayor de 60 años. Revista Cubana de Estomatología.2010; 47(1)105-114.

14. García-Alpízar B, Capote-Valladares M, MoralesMontes-de-Oca T. Prótesis totales y lesiones bucales en adultos mayores institucionalizados. Revista Finlay [revista en Internet]. 2012 [Citado 2020 febrero 2]; 2(1):[aprox. 12 p.]. Disponible en:http://www.revfinlay.sld.cu/index.php/finlay/arti cle/view/99

15. Instituto Nacional de Estadística e Informática. Situación de la Población Adulta Mayor. INFORME TÉCNICO $\mathrm{N}^{\circ} 1$ Marzo 2018.Disponible en: https://www.inei.gob.pe/media/MenuRecursivo/bo letines/01-informe-tecnico-n02_adulto_enefeb_mar2018.pdf

16. A healthy lifestyle. [Internet] World Health Organization Regional Office for Europe; 2020 [Citado 2020 marzo 11] . Disponible en: https://www.euro.who.int/en/health-topics/disease -prevention/nutrition/a-healthy-lifestyle

17. HEARTS Technical package for cardiovascular disease management inprimary health care: healthy-lifestyle counselling; Geneva:World Health Organization; 2018. Disponible en: https://apps.who.int/iris/bitstream/handle/10665/2 60422/WHO-NMH-NVI-18.1eng.pdf?sequence $=1$

18. Li Y, Schoufour J, Wang DD, Dhana K, Pan A, Liu $X$ et al.Healthy lifestyle and life experiency free of cáncer, cardiovascular disease and type 2 diabetes: prospective cohort study. BMJ 2020;368:1660 doi: 10.1136/bmj.I6669

19. Alzate-Urrea S, Agudelo-Suárez AA, López-
Vergel F, López-Orozco C, Espinosa-Herrera E, Posada López A. Calidad de vida y salud bucal: Perspectiva de adultos mayores atendidos en la red hospitalaria pública Rev. Gerenc. Polít. Salud. 2015; 14(29): 83-96.

http://dx.doi.org/10.11144/Javeriana.rgyps1429.cbsv

20. Meneses Gómez E.J. Salud bucodental y calidad de vida oral en los mayores. [Tesis doctoral]. Madrid: Universidad Complutense de Madrid; 2010.

21. Varela Pinedo Luis F. Salud y calidad de vida en el adulto mayor. Rev. Perú. med. exp. salud publica [Internet]. 2016 Abr [citado 2020 feb 04] ;33( 2):199-201.Disponible en: http://www.scielo.org.pe/scielo.php?script=sci_art t ext\&pid=S1726-46342016000200001\&lng=es. http://dx.doi.org/10.17843/rpmesp.2016.332.2196

22. Bardales Carreño, L.G. Condiciones clínicas bucales más prevalentes y necesidad de rehabilitación bucal en 150 pacientes adultos mayores en el Albergue Central Ignacia R. Vda. de Canevaro. Lima 2003. [Tesis para optar el Título de Cirujano Dentista]. Universidad Inca Garcilaso de la Vega. Lima; 2003.

23. Singla N, Acharya s, Singla R, Nayak P, The impact of Lifestyle on Dental Caries of Adult Patients in Udupi District: A Cross-sectional Study. J Int Soc Prevent Communit Dent 2020;10:189-95.

24. Rojas González C, Segovia Chamorro J, Raccoursier Schwerter V, Godoy Olave J, Lopetegui Buschmann MS. Estado periodontal y necesidad de tratamiento en pacientes GES 60 años de Villa Alemana. Rev. Clin. Periodoncia Implantol. Rehabil. Oral [Internet]. 2010 Ago [Citado 2020 feb 19] ; 3( 2 ): 86-89. Disponible en: https://scielo.conicyt.cl/scielo.php?script=sci_artt ext\&pid=S0719-01072010000200005\&Ing=es.

25. Pérez Hernández L. Salud periodontal en gerontes de casas de abuelos en Pinar del Río.Rev. Ciencias Médicas. Marzo-abril, 2015; 19(2):195- 205

26. Naimi-Akbar A, Kjellström B, Rydén $L$, Rathnayake N, Klinge B, Gustafsson A \& Buhlin $\mathrm{K}$. Attitudes and lifestyle factors in relation to oral health and dental care in Sweden: a crosssectional study. Acta Odontologica Scandinavica. 2019;77(4): 282- $289 . \quad$ DOI: 10.1080/00016357.2018.1539238

27. Carvajal Paola. Enfermedades periodontales como un problema de salud pública: el desafío del nivel primario de atención en salud. Rev. Clin. Periodoncia Implantol. Rehabil. Oral [Internet]. 2016 Ago [Citado 2020 Mar 2]; 9(2): 177-183.Disponible en: https://scielo.conicyt.cl/scielo .php?script=sci_arttext\&pid=S071901072016000200016\&lng=es.

$\begin{array}{ll}\text { Rosa Elizabeth Ysla Cheé } & \text { https://orcid.org/ } \\ \text { rosayslache@yahoo.es } & \text { (1) http://orcid.org/0000-0003-5497-6231 } \\ \begin{array}{l}\text { María Pareja Vásquez } \\ \text { mparejav@usmp.pe }\end{array} & \text { (c) https://orcid.org/0000-0001-9805-8785 } \\ \begin{array}{l}\text { Jaime Carlos Porras Cerrón } \\ \text { jaimepc@lamolina.edu.pe }\end{array} & \end{array}$

